\title{
Prävention, Patientenrechte und Rehabilitation im Koalitionsvertrag
}

ROLF ROSENBROCK, CLAUDIA ZINKE

Prof. Dr. Rolf Rosenbrock war von 1999 - 2009 Mitglied im Sachverständigenrat zur Begutachtung der Entwicklung im Gesundheitswesen (SVR-G) und ist seit 2012 Vorsitzender des Paritätischen Wohlfahrtsverbandes Gesamtverband in Berlin

Claudia Zinke ist Leiterin der Abteilung Gesundheit und Rehabilitation beim Paritätischen Wohlfahrtsverband Gesamtverband in Berlin

\author{
Der Vergleich zwischen den Plänen für \\ Prävention, Patientenrechte und Rehabilitation im \\ Koalitionsvertrag ergibt ein gemischtes Bild: während \\ für die nicht-medizinische Primärprävention und \\ Gesundheitsförderung mit dem vierten Anlauf zu einem \\ Bundespräventionsgesetz ein relativ großes Projekt \\ ansteht und im Vertrag zwar lückenhaft, aber mit einer \\ im Wesentlichen realistischen Regulierungsperspektive \\ vorgestellt wird, scheint es den Vertragspartnern bei \\ den Themen Patientenrechte und Rehabilitation an \\ Problembewusstsein, Mut und Phantasie eher gefehlt \\ zu haben: hier gibt es keine Überraschungen, bekannte \\ und behebbare Probleme werden nicht angegangen.
}

\section{Primärprävention und Gesundheitsförderung}

Primärprävention und Gesundheitsförderung spielen trotz überzeugender Argumente für die Notwendigkeit und starker Evidenz für die Wirksamkeit in der deutschen Gesundheitspolitik eine nach wie vor beklagenswert geringe Rolle - vor allem mit Blick auf die große und wachsende sozial bedingte Ungleichheit von Gesundheitschancen. Es passiert nach wie vor viel zu wenig, und das Wenige, was gemacht wird, ist meistens wissenschaftlich überholte Verhaltensprävention mit viel Gesundheitsförderungs-Rhetorik und wenig Nachhaltigkeit. Vermehrt werden müssen nicht-medizinische Interventionen und Strategien der Primärprävention, die insbesondere bei sozial benachteiligten Menschen v. a. chronisch-degenerative Erkrankungen verhüten oder zeitlich verschieben. Das Mittel der Wahl dafür sind partizipativ angelegte und die Lebenswelt/das Setting physisch und sozial verändernde Interventionen. Sie sind nach wie vor die Ausnahme.
Die Delegation der Primärprävention an die gesetzliche Krankenversicherung GKV (im $\$ 20$ SGB V, zunächst ab 1989, verstärkt ab 2000) war zunächst einmal die Nutzung der politischen Gelegenheit (,policy window ${ }^{\mathrm{C}}$ ), das Thema und die damit verbundenen Aktivitäten überhaupt irgendwo zu institutionalisieren. Prävention durch GKV erwies sich auch - positiv wie negativ - als geeignetes Lernfeld. Positiv, weil die GKV zumindest in der betrieblichen Gesundheitsförderung und vereinzelt auch in KiTas, Schulen und Stadtteilen state oft he art-Interventionen förderte und fördert. Positiv auch, weil die GKV sich recht früh mit dem Problem angemessener Qualitätssicherung befasste. Auf dieser Basis steht Deutschland heute in Bezug auf die gesundheitsförderliche Gestaltung von Setting/Lebenswelten sowie im Hinblick auf Qualitätsentwicklung in der Prävention auch im europäischen Vergleich recht gut da. Negativ waren die Erfahrungen, weil der Gesetzgeber die Kassen zugleich mit ihrer Beauftragung mit für die Prävention ungeeigneten und kontraproduktiven ökonomischen Anreize versah: 
Unter Kassenkonkurrenz und bei unzureichendem Risikostrukturausgleich können die Kassen kein ökonomisches Interesse daran haben, besonders attraktiv für sozial benachteiligte Menschen zu sein, da diese nicht nur unterdurchschnittliche Beiträge zahlen, sondern auch überdurchschnittlich häufig und

\section{Die Serie der gescheiterten Anläufe für ein Präventionsgesetz muss mit einem legislativen Neuanfang durchbrochen werden.}

ler Sozialversicherungsträger sowie der Länder und Kommunen werden über verpflichtende Rahmenvereinbarungen analog der Regelungen zur Förderung der Zahngesundheit ( $\mathbb{S} 21$ SGB V) und von Schutzimpfungen ( $\mathbb{S} 20 \mathrm{~d}$ Abs. 3 SGB V) auf Landesebene verbessert. Dabei sind bundesweit einheitliche Gesundheitsziele und Vorgaben zur Qualität und Evaluation zu berücksichtigen. Länderpräventionsansätze werden einbezogen." (Abschnitt 2.4)

Auf Basis und ohne Widerspruch zu diesen dürren Formulierungen lässt sich ein sehr gutes, europaweit vorbildliches Präventionsgesetz schwer krank sind. Der Ertrag erfolgreicher Prävention ist der einzelnen Kasse wegen der freien Kassenwahl auch nicht sicher. Hingegen haben die Kassen unter diesen Anreizen ein starkes Interesse daran, Präventionsangebote zu machen, die den Bedürfnissen der - weniger kranken und beitragsstärkeren - Mittelschichten entsprechen. Noch heute geben die Kassen infolgedessen mehr als $80 \%$ der Ressourcen nach $\$ 20$ SGB V für Kurse zur Verhaltensänderung (,individueller Ansatz') aus, mit denen Menschen aus den unteren Sozialschichten kaum erreicht werden und deren nachhaltige Wirksamkeit gering ist.

Deshalb gibt es seit Beginn der 2000er Jahre die Forderung nach einem neuen rechtlichen Rahmen. Die bisherigen drei Anläufe (2005, 2008, 2013) waren - gemessen an den Anforderungen und den Möglichkeiten - sämtlich suboptimal und wurden von Mal zu Mal schlechter. Die Ablehnung des 2013er Entwurfs durch den Bundesrat im September 2013 war verdienstvoll, weil die Reparatur all der gesundheitswissenschaftlichen und regulativen Fehler dieses Produkts weit mehr legislative Arbeit bedeutet hätte als die Produktion eines neuen Entwurfs.

Die Serie gescheiterter Anläufe muss mit einem legislativen Neuanfang durchbrochen werden. Den verspricht nun der Koalitionsvertrag: „Wir werden noch 2014 ein Präventionsgesetz verabschieden, das insbesondere die Prävention und Gesundheitsförderung in Lebenswelten wie KiTa, Schule, Betrieb und Pflegeheim und die betriebliche Gesundheitsförderung stärkt und alle Sozialversicherungsträger einbezieht. Die Kooperation und Koordination al- entwickeln, aber eine Garantie für ein gutes Gesetz sind diese Sätze nicht.

Die Qualität des Gesetzes wird sich daran entscheiden und messen lassen, ob und inwieweit die folgenden Punkte berücksichtigt werden:

Seit Jahrzehnten fristet nicht-medizinische Prävention und Gesundheitsförderung ein Schicksal als Thema von weitgehend konsequenzlosen Sonntagsreden und symbolischen Aktionen. Notwendig ist, dass die damit gemeinten Strategien und Maßnahmen legislativ sichtbar in den gleichen Rang gehoben werden, wie medizinische, pflegerische und rehabilitative Interventionen. Dazu bedarf es eines eigenständigen Gesetzes, am besten als eigenes Buch im SGB-Kanon.

$\mathrm{Zu}$ regeln ist der gesamte Bereich öffentlich geförderter Prävention und Gesundheitsförderung, und zwar von allen Sozialversicherungen, dem Öffentlichen Gesundheitsdienst (ÖGD) und anderen kommunalen Stellen sowie von Länder- und Bundesprogrammen. Dabei wird es zu Abgrenzungsproblemen kommen, z. B. zu Programmen der Kriminalitätsprävention oder der Arbeit gegen Fremdenfeindlichkeit; denn diese arbeiten oft mit der gleichen Methodik, zielen auf die Stärkung der gleichen personalen Faktoren und wollen auch dieselben Zielgruppen erreichen. Ob dieser Sachverhalt vernünftig geregelt wird, hängt von der Reichweite und der Verbindlichkeit der angezielten Rahmenvereinbarung(en) ab.

Die epidemiologische Situation und ihre Dynamik erfordern, dass das Regelwerk - wie der bestehende $\mathbb{S} 20$ SGB V - an dem Ziel ausgerichtet wird, den allgemeinen Gesundheitszustand der
Bevölkerung zu verbessern und dabei insbesondere einen Beitrag zur Verminderung sozial bedingter Ungleichheit von Gesundheitschancen zu erbringen. Ein Bezug darauf findet sich im Koalitionsvertrag erstaunlicherweise nicht.

Zur Bündelung der praktischen und wissenschaftlichen Kräfte wie auch zur vergleichenden Ergebnismessung sollen sich die Interventionen auf Präventionsziele und nicht - wie der Koalitionsvertrag missverständlich formuliert: Gesundheitsziele - beziehen. Während Gesundheitsziele auch Versorgungsziele enthalten (und in der Arbeit von gesundheitsziele.de tun sie dies auch), ist die Festlegung von Präventionszielen eine eigenständige und fachlich nicht zu unterschätzende Aufgabe, bei der es z. B. auch um die Festlegung von Zielindikatoren (ähnlich den Markern in der Medizin) mit spezifischen Problemen der Erhebung und der Messbarkeit geht.

Prävention bedarf wie jede gesundheitsbezogene Intervention einer verbindlichen Qualitätssicherung - das Ziel ist die evidence based prevention. Für diesen Weg hat es sich als nützlich erwiesen, zwischen der - in aller Regel partizipativ zu gestaltenden - Qualitätsentwicklung einerseits und der Ergebnisbestimmung bzw. -Messung zu unterscheiden. Der Gesetzentwurf von 2005 enthielt diesbezüglich vorbildlich differenzierte Anforderungen an einerseits verhaltensbezogene und Setting/ Lebenswelt-Interventionen andererseits. Auf Basis ihrer jahrelangen Vorarbeiten hat sich insbesondere die Bundeszentrale für gesundheitliche Aufklärung dafür qualifiziert, als Sachwalterin der Entwicklung und Anwendung deutschlandweit verbindlicher Vorgaben für die Qualität der Prävention zu fungieren.

Die auch seit Jahrzehnten von der Weltgesuntheitsorganisation (WHO) propagierten Interventionen zur partizipativen Gestaltung gesundheitsförderlicher Lebenswelten/Settings versprechen v. a. im Hinblick auf die Erreichbarkeit und die Einbeziehung sozial benachteiligter Gruppen den größten Ertrag. Zur Vermeidung von bequemem Etikettenschwindel müssen sie aber abgegrenzt werden von Interventionen, die die Lebenswelt/das Setting lediglich als Einflugschneise und Ablageort klassischer Gesundheitsbotschaften nutzen und weder die Potenziale direkter Partizipation nutzen noch das Setting/die Lebenswelt 
selbst verändern. Diese Interventionen aber sind komplex, störanfällig, schwierig zu steuern, sie brauchen einen langen Atem und hohen Einsatz. Wenn dem Versprechen des Koalitionsvertrages genügt werden soll, muss das Gesetz nicht nur klare Definitionen und Kriterien sondern auch Regelungen, zum Beispiel in Form von Quotierungen enthalten, um der Tendenz zum Einfachen, Harmlosen und Wirkungsarmen entgegenzuwirken. Der Entwurf des Präventionsgesetzes von 2005 war diesbezüglich allen nachfolgenden Anläufen überlegen.

Entwurfsfassungen der Koalitionsvereinbarungen enthielten noch feste EuroBeträge der GKV für die Prävention einschließlich des vorgesehenen Zuwachses. Dass der Koalitionsvertrag diese Vorgaben nicht mehr enthält, muss für die Finanzierung nichts Negatives bedeuten. Denn mit dem geplanten Gesetz soll die Finanzierung ja auf mehr Akteure verteilt und auf eine neue Grundlage gestellt werden. Natürlich ist der Finanzbedarf wesentlich höher als die heute eingesetzten Mittel, wenn man bedenkt, dass es in Deutschland mehr als 100.000 KiTas und Schulen, einige 10.000 Pflege- und Alteneinrichtungen, ca. 2 Mio. Betriebe mit mehr als einem Beschäftigtem, einige tausende soziale Brennpunkte etc. etc. gibt - alles Lebenswelten, in denen mit Sinn und Nutzen interveniert werden könnte und sollte. Das Interventionsfeld Kommune, Stadtteil, Sozialer Brennpunkt fehlt allerdings gegen alle Logik in der Aufzählung des Koalitionsvertrages; in den Ausführungen zum Programm, Soziale Stadt' (Abschnitt 4. 2) hingegen fehlt der Hinweis auf die Verbindung dieses Themas mit der Setting-bezogenen Prävention. Die Betriebliche Gesundheitsförderung findet sich dafür nicht nur im Absatz zum Präventionsgesetz, sondern auch noch einmal ausführlich im Abschnitt, Gute Arbeit' (2.2). Es fehlt also weder an Interventionsfeldern noch an Interventionsmethoden. Allerdings gibt es derzeit noch keineswegs hinreichend qualifizierte Akteure in ausreichender Zahl, um diese Aufgaben sofort angehen zu können. Das kommende Präventionsgesetz wird deshalb auch die Basis sein, auf der sich solche Leistungserbringer v. a. aus der Zivilgesellschaft - in größerer Anzahl entwickeln und qualifi- zieren können und sollen. Deshalb sind die wichtigsten Fragen der Finanzierung die Kriterien, Mechanismen und Träger ihres Aufwuchses.

Niemand in Deutschland weiß, wie viel Geld derzeit hierzulande von Sozialversicherungen, Gemeinden, Ländern und dem Bund für nicht-medizinische Primärprävention und Gesundheitsförderung ausgegeben wird. Das ließe sich herausfinden. Damit wäre der Betrag be- haus. Mit dieser neuen Bürokratie soll der v. a. als Ungerechtigkeit empfundenen und im Kern unethischen Bevorzugung von privat Versicherten durch gegenwartsnähere Terminvergabe entgegengewirkt werden. Keine Vorschläge enthält der Koalitionsvertrag zur Veränderung des zugrunde liegenden Anreizes aus der um bis zu $100 \%$ differierenden Honorarhöhe zwischen der Gebührenverordnung für Ärzte (GOÄ) und dem Einheitlichen Bewertungsmaßstab (EBM) für privat und gesetzlich Versicherte. Absehbar ist, dass die Terminvergabestellen von Menschen mit geringerer formaler Bildung unterdurchschnittlich häufig in Anspruch genommen werden.

- GKV-Versicherte können auf Kosten ihrer Kasse eine Zweitmeinung einholen. Neu daran ist eigentlich nur, dass sie darauf von den behandelnden Ärzten hingewiesen werden müssen, unverständlich (und überflüssig) ist die Beschränkung auf ,mengenanfällige planbare Leistungen.

- Zu begrüßen sind der vorgesehene Rechtsanspruch auf Pflege- bzw. Familienpflegezeit zur Ermöglichung der Vereinbarkeit von Pflege und Beruf sowie die Vorgabe einer Lohnersatzleistung für bis zu zehn Tage für pflegende Angehörige, die ein neues Pflegearrangement organisieren müssen. Wie beim Pflegetelefon und der weiteren Entwicklung der Pflegestützpunkte hängt die Wirksamkeit der löblichen Absichten von der Ausgestaltung ab.

\section{Patientenrechte}

„Im Zentrum unserer Gesundheitspolitik stehen die Patientinnen und Patienten“, so die ersten Worte des Kapitels 2.4. ,Gesundheit und Pflege', und diesem Ziel sollen auch all die Neuerungen aus dem Koalitionsvertrag dienen. Ob und in welchem Ausmaß sie dies oder etwas Anderes tun, wird man sehen. Weil aber das Versorgungssystem, die Versicherungen und die staatliche Aufsicht diese Haltung nicht von selber hervorbringen, braucht es besondere Rechte für Versicherte und Patienten. In dieser Hinsicht verspricht der Koalitionsvertrag nicht allzuviel:

- Für GKV-Versicherte werden bei den Kassenärztlichen Vereinigungen (KV) Terminservice-Stellen eingerichtet, die ihnen einen Facharzttermin in der Regel innerhalb von vier Wochen sichern sollen - ambulant oder im Kranken-
In den Entscheidungsgremien des Medizinischen Dienstes der Krankenkassen (MDK) sollen künftig Vertreter der Pflegebedürftigen und ihrer Angehörigen sowie der Pflegeberufe stimmberechtigt vertreten sein. Das ist neu und verspricht - im Lichte der Erfahrungen mit der PatientInnenvertretung im Gemeinsamer Bundesausschuss (G-BA) und in den Landesausschüssen - eine interessante Entwicklung. Ob sie den MDK so verändert, dass die Forderungen nach Auflösung des zugrunde liegenden Interessenkonfliktes durch institutionelle Loslösung des MDK von den Kassen verstummen, erscheint allerdings wenig wahrscheinlich.

- Löblich auch, dass Selbsthilfe-Arbeit (im Abschnitt „Pflege“) Erwähnung findet und besser gebündelt und 
vernetzt werden soll. Konzepte, die auf eine verbindliche Bündelung der Kräfte und eine adäquate Strukturförderung der Patienten- und Selbsthilfeorganisationen abzielen und damit der Sichtweise der Patienten und Nutzer ein insgesamt stärkeres Gewicht im Gesundheitskorporatismus verleihen würden, haben keinen Eingang in den Koalitionsvertrag gefunden.

Im Hinblick auf Versicherten- und Patientenrechte ist der Koalitionsvertrag weit entfernt von einem ,mehr Demokratie wagen', und keine der angesprochenen Probleme wird auf dem im Vertrag bezeichneten Weg tatsächlich zu lösen sein. Das gilt auch für die nicht im Vertrag erwähnten Baustellen der Patientenrechte: Die Beteiligungsrechte der Patientenver-

\section{Patientenrechte standen offensichtlich nicht im Focus des Interesses der Verhandlungs- und Vertragspartner.}

treter im G-BA (Stimmrecht zumindest in Verfahrensfragen; Verstärkung der fachlichen und organisatorischen Unterstützung) werden nicht angesprochen. Für das erst 2013 verabschiedete Patientenrechtegesetz ist - trotz überparteilichem Konsens über die Notwendigkeit - immer noch kein Härtefallfonds vorgesehen, und auch die mit der UN-Konvention über die Rechte behinderter Menschen (UN-BRK) unverträgliche Rechtskonstruktion der ,Entscheidungsunfähigkeit ${ }^{\star}$ im Gesetz findet keine Erwähnung. Das gilt auch für die zunehmend dringlich diskutierte Frage, ob die erfolgreich arbeitende ,Unabhängige Patientenberatung Deutschland' (UPD) nach $\mathbb{S} 65 \mathrm{~b}$ SGB V beim GKV-Spitzenverband anreiz- und interessenmäßig korrekt angesiedelt ist.

Zusammenfassender Befund: Patientenrechte standen offensichtlich nicht im Focus des Interesses der Verhandlungsund Vertragspartner. Wichtige Fragen sind überhaupt nicht angesprochen, die im Vertrag vorgesehenen Innovationen werden die angesprochenen Probleme allenfalls verkleinern, aber nicht lösen, in manchen Fällen auch komplizierter machen.

\section{Rehabilitation}

Der Reformbedarf in der und für die Rehabilitation ist beachtlich. Steigende Lebenserwartung, längere Lebensarbeitszeit, veränderte Belastungen, Verschiebungen im Krankheitsgeschehen, schnellere Entlassungen aus dem Akutkrankenhaus, bedingt durch die diagnosebezogenen Fallgruppen (DRG), und die Verpflichtungen aus der UN-BRK zur Rehabilitation für behinderte Menschen (auch z. B. mit Dememz) führen zu wachsendem Bedarf. Die Ausgaben für Reha sind seit 1997 budgetiert (,gedeckelt'); die Schere zwischen Bedarf und Bewilligungen öffnet sich; die Bewilligungsquoten sanken z.B. in der für die Reha von Beschäftigten zuständigen gesetzliche Rentenversicherung auf mittlerweile 64,3\% (2011), die Fallzahlen hatten auch 2012 noch nicht wieder das Niveau der Jahrhundertwende erreicht. Nach wie vor ,hakt es' auch in der Kooperation zwischen den Reha-Trägern, die gemeinsamen örtlichen Servicestellen haben nicht die gewünschte Transparenz und Erleichterung für Antragsteller erbracht, die Entwicklung zur gesundheitswissenschaftlich gewünschten Ambulantisierung verläuft nur schleppend etc.

Gemessen an diesen Herausforderungen sind die Vorhaben im Koalitionsvertrag äußerst bescheiden: zu den Kooperations- und Schnittstellen-Problemen findet sich kein Wort, von einer besseren Orientierung an den Bedürfnissen der Antragsteller bzw. Rehabilitanden ist nicht die Rede. Zur Minderung der Finanzprobleme wird vorrangig auf andere Akteure verwiesen: durch mehr betriebliche Prävention und bessere Krankenversorgung soll der Bedarf in Grenzen gehalten werden; was an Reha-Bedarf übrig bleibt, soll im Rahmen eines angepassten Reha-Budgets abgesichert werden. Der ,Deckel' soll also angehoben werden, für das Ausmaß der Anhebung wurde die schicke Leervokabel ,demografiefest ${ }^{\circ}$ gewählt. Zudem soll geprüft werden, ob die Pflegeversicherung an der bislang von der GKV getragenen Finanzierung der geriatrischen Reha beteiligt werden soll. Das ist zwar unter Anreizgesichtspunkten nicht unplausibel, verschiebt nur, aber löst nicht die insgesamt beachtlichen Finanzierungsprobleme der Rehabilitation.
Zusammenfassender Befund: Der Koalitionsvertrag macht im oft in seiner Wichtigkeit unterschätztem Bereich der Rehabilitation nicht den Eindruck, als wolle die große Koalition hier einen Schwerpunkt setzen oder sei von dem Willen getragen, sich dem Gesamtspektrum der aufgelaufenen Probleme zu stellen. Das kann sich im Verlauf der nächsten vier Jahre rächen.

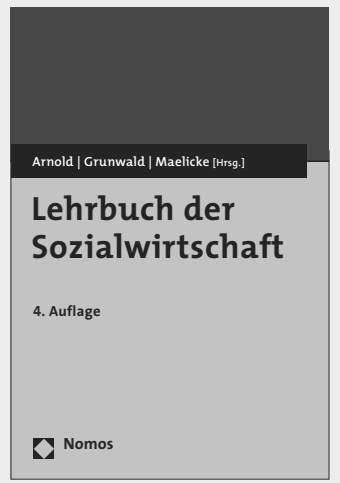

\section{Lehrbuch der Sozialwirtschaft}

Herausgegeben von Ulli Arnold, Klaus Grunwald und Bernd Maelicke

4. Auflage 2014, ca. 900 S., brosch., ca. 49,- $€$

ISBN 978-3-8329-7819-8

Erscheint ca. März 2014

Die rapide Veränderung der politischen und strukturellen Rahmenbedingungen für die Sozialwirtschaft erfordert grundlegende Änderungen in der Ausbildung und Weiterbildung von Führungskräften sozialer Dienstleistungsorganisationen. Die vollständig aktualisierte und neu bearbeitete 4. Auflage vermittelt Basiswissen und aktuelle Informationen zu den wichtigsten Themen.

www.nomos-shop.de/19645 Nomos 